\title{
Resuscitation with Intact Placental Circulation in a Preterm Infant with Hydrops Fetalis
}

\author{
Simone Pratesi, MD, PhD ${ }^{1} \quad$ luri Corsini, MD ${ }^{1} \quad$ Caterina Coviello, MD ${ }^{1} \quad$ Silvia Perugi, MD ${ }^{1}$ Carlo Dani, MD \\ ${ }^{1}$ Neonatology and Intensive Care Unit, Department of Maternal and \\ Child Health, Careggi University Hospital, Florence, Italy \\ Am J Perinatol Rep 2017;7:e28-e30. \\ Address for correspondence Simone Pratesi, MD, PhD, Neonatology \\ and Intensive Care Unit, Department of Maternal and Child Health, \\ Careggi University Hospital, Largo Brambilla 1, 50139 Florence, Italy \\ (e-mail: simone.pratesi@unifi.it).
}

\begin{abstract}
A preterm infant with prenatal diagnosis of hydrops fetalis was spontaneously delivered at 30 weeks of gestational age in a tertiary level hospital. Prenatal echography pointed out severe bilateral pleural effusions and diffused subcutaneous edema. A neonatologist team, alerted at the expulsion stage of labor, assisted the neonate immediately after birth and bilateral hydrothorax was drained with intact placental circulation to

\section{Keywords}

- hydrops fetalis

- neonatal resuscitation

- delayed cord clamping

- bedside resuscitation avoid a nonrespiratory period and its possible detrimental hemodynamic effects. The newborn was well stabilized in the delivery room before cutting the umbilical cord and starting mechanical ventilation. Unfortunately, our patient died due to refractory respiratory failure on the fourth day of life. However, the intact placental circulation procedure was performed without adverse effects to the infant and might represent a promising option in addition to other resuscitation procedures for the management of this type of patient.
\end{abstract}

Hydrops fetalis is a rare, potentially lethal pathology occurring in the fetal and neonatal period, with a survival rate ranging from 27 to $36 \%^{1,2}$ It is a condition of excessive fetal fluid accumulation in at least two serous cavities (i.e., abdomen, pleura, pericardium) or in body tissue (subcutaneous edema). Nonimmune hydrops fetalis constitutes up to $90 \%$ of all cases of hydrops fetalis, ${ }^{3}$ and may be of different etiologies, such as cardiovascular disorders (21.7\%), chromosome imbalance (13.4\%), hematologic abnormalities (10.4\%), infections (6.7\%), intrathoracic masses (6.0\%), lymph vessel dysplasias (5.7\%), twin-to-twin transfusion syndrome and placental causes (5.6\%), syndromes (4.4\%), urinary tract malformations (2.3\%), inborn metabolic errors (1.1\%), extrathoracic tumors (0.7\%), gastrointestinal disorders (0.5\%), miscellaneous (3.7\%), and idiopathic causes (17.8\%). ${ }^{3}$ A severe bilateral pleural effusion can compress fetal lungs and impede lung expansion at delivery despite the support of positivepressure ventilation. Thus, an immediate postnatal thoracentesis is needed to drain the intrapleural fluid to permit proper lung ventilation and gas exchange. Currently, the resuscitation of preterm infants with antenatal hydrothorax

received

November 13, 2016 accepted after revision December 21, 2016
$10.1055 / \mathrm{s}-0037-1598200$ ISSN 2157-6998. includes umbilical cord cutting, a rapid transfer of the infant to the radiant infant warmer, his/her intubation and mechanical ventilation, and then thoracentesis. Therefore, the time from the cord cutting and the start of effective ventilation may entail a period of nonrespiration during which the newborn, especially if preterm, can experience hypoxemia and be at risk of asphyxia and cerebral hemodynamic impairment. Recently, it has been suggested that delaying cord clamping until after the start of breathing by maintaining the placental circulation intact would be more physiologically and hemodynamically advantageous for the newborn in comparison with the current management that recommends first cord clamping/cutting and then commencement of positive-pressure ventilation within 60 seconds of life. Thus, we report a novel approach to neonatal resuscitation in the delivery room in a preterm infant with an antenatal diagnosis of severe bilateral hydrothorax in whom postnatal thoracenteses were performed before the cord clamping with intact placental circulation to limit the risk of ineffective pulmonary gas exchanges due to the application of positive-pressure ventilation to unexpanded lungs.

Copyright @ 2017 by Thieme Medical Publishers, Inc., 333 Seventh Avenue, New York, NY 10001, USA. Tel: +1(212) 584-4662.

\section{License terms}

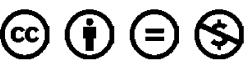


A 20-year old primipara vaginally delivered a $30^{+5}$ weeks' gestation male newborn with a birth weight of 2,300 $\mathrm{g}$ after a pregnancy complicated by polyhydramnios, fetal massive skin edema, and severe bilateral pleural effusion with collapsed lungs diagnosed at 24 weeks of gestation. The mother was referred to our center only 2 days before delivery. The worsening of umbilical and fetal flows made in uterus placement of pleural drainage dangerous. Thus, in agreement with parents, it was decided to induce labor pharmacologically after antenatal steroid prophylaxis.

After informed parental consent, the neonatal resuscitation team assisted the newborn at delivery, using a special neonatal resuscitation bed (LifeStart, Inditherm Medical, Rotherham, United Kingdom) on which he was placed maintaining intact his 52-cm-long umbilical cord for approximately 10 minutes. At birth the newborn was cyanotic, apneic, severely bradycardic, hypotonic, and presented a severe and diffused skin edema. After suctioning of the mouth and nose, one neonatologist started mask ventilation using a T-piece (peak inspiratory pressure $[\mathrm{PIP}]=20 \mathrm{~cm} \mathrm{H}_{2} \mathrm{O}$ and positive end-expiratory pressure [PEEP] $5 \mathrm{~cm} \mathrm{H}_{2} \mathrm{O}$ ) with $30 \%$ inspired oxygen (increased to $100 \%$ during the first minute of life on the basis of $\mathrm{SpO}_{2}$ ), whereas two other neonatologists performed an immediate bilateral thoracentesis via 19G intravenous catheter through the fourth intercostal space, while placental circulation was maintained intact. Overall, greater than $200 \mathrm{~mL}$ of transparent and citrin pleural fluid was drained. The heart rate increased above 100 beats/min during the second minute of life whereas $\mathrm{SpO}_{2}$ progressively increased from the initial value of 50 to $90 \%$ at 5 minutes of life (see - Video 1). At 5 minutes of life the newborn was pink, spontaneous breathing began, $\mathrm{SpO}_{2}$ was $97 \%$, and heart rate was 150 beats/min. The infant was then intubated for starting mechanical ventilation ( $\mathrm{PIP}=20 \mathrm{~cm} \mathrm{H}_{2} \mathrm{O}$ and PEEP $5 \mathrm{~cm} \mathrm{H}_{2} \mathrm{O}$, respiratory rate 60 breaths/min) as pleural effusions were likely to relapse and surfactant administration had to be performed. The umbilical cord was clamped at approximately 10 minutes of life without any apparent adverse effects. Our patient was treated with high-frequency oscillatory ventilation (HFOV), $100 \%$ inspired oxygen, three doses of surfactant for severe respiratory failure, and inhaled nitric oxide for an associated pulmonary hypertension in the neonatal intensive care unit (NICU). Bilateral hydrothorax quickly relapsed and bilateral pleural tube drainages were placed for continuous aspiration. His pleural fluid was found with $97 \%$ lymphocyte cell count, suggesting the diagnosis of congenital chylothorax. ${ }^{4}$ Therefore, at 20 hours of life we started treatment with intravenous octreotide $(1 \mu \mathrm{g} / \mathrm{kg} / \mathrm{h}$ that was progressively increased to $5 \mu \mathrm{g} / \mathrm{kg} / \mathrm{h}$ ) that allowed a significant reduction in pleural effusions after 48 hours of therapy. It is noteworthy that in the first 3 days of life the newborn's weight decreased by $1,000 \mathrm{~g}$. Unfortunately, in the fourth day of life his clinical conditions were further complicated by right pneumothorax, and ultimately the infant died due to refractory respiratory failure.

To our knowledge, this is the first case report on the resuscitation with intact placental circulation of a preterm infant with hydrops fetalis delivered vaginally. Few case

\section{Video 1}

Neonantal resuscitation with intact placental circulation in a preterm infant with hydrops fetalis. Online contentincluding videosequences viewable at: www.thieme-connect.com/products/ ejournals/html/10.1055/s-0037-1598200.

reports have been previously published on extrauterine intrapartum treatment (EXIT) in isolated unilateral or bilateral fetal hydrothorax delivered at term by cesarean section. ${ }^{5-8}$ According to the international guidelines on neonatal resuscitation, infants who need resuscitation at birth should have immediate cord clamping and positive-pressure ventilation must start within 60 seconds of life. ${ }^{9}$ We decided to resuscitate our infant while maintaining intact the placental circulation because we thought that it could be advantageous for the newborn. In fact, during fetal life oxygenated blood coming from the placenta through the umbilical vein mainly reaches the left ventricle of the heart through the foramen ovale. The placenta guarantees the left ventricle preload and maintains an adequate cardiac output toward the aorta and epiaortic vessels. On the other hand, if the cord is clamped immediately after birth, the left ventricle suddenly loses its filling source and its preload becomes fully dependent on pulmonary vein blood returning from the lungs. Pulmonary blood flow increases slowly after birth when the newborn starts to breathe, inducing a decline in pulmonary vascular resistance. Thus, during the so-called nonrespiring period between the cord clamping and the start of spontaneous breathing, the left ventricle is no longer receiving oxygenated blood from the placenta and not yet from the lungs, so systemic cardiac output may be decreased and hemodynamic fluctuations may be associated with reduced cerebral oxygenation, as previously demonstrated in animal models. ${ }^{10,11}$ Otherwise, if the cord is not clamped, blood coming from the placenta through the umbilical vein continues to fill the left ventricle, allowing the onset of the newborn's breathing to increase pulmonary blood flow and venous circulation from the lungs. ${ }^{12,13}$ Thus, maintaining intact the placental circulation by delaying cord clamping until after the start of breathing could preserve left ventricle preload-promoting hemodynamic stability and possibly helping to decrease the occurrence of intraventricular hemorrhage in the preterm infant without adverse effects, as suggested by a recent meta-analysis. ${ }^{14}$ On the basis of previous considerations, a preterm newborn with hydrops fetalis may be the ideal candidate for resuscitation with intact placental circulation because his gas exchanges can be continuously guaranteed by the placenta and left ventricle preload and cardiac output maintained by umbilical vein flow until bilateral thoracentesis and simultaneous positive pressure ventilation allow lung recruitment and the start of proper pulmonary function. Moreover, vaginally delivered newborns benefit from a longer and greater placental blood transfusion than C-section-delivered babies. ${ }^{15}$ In conclusion, we report the case of a preterm infant with hydrops fetalis who was resuscitated in the delivery room with intact placental circulation to avoid the 
nonrespiratory period and its possible detrimental hemodynamic effects. The procedure occurred without adverse effects and might represent a promising option-in addition to other resuscitation procedures-for the management of these patients.

\section{References}

1 Santo S, Mansour S, Thilaganathan B, et al. Prenatal diagnosis of non-immune hydrops fetalis: what do we tell the parents? Prenat Diagn 2011;31(02):186-195

2 Ota S, Sahara J, Mabuchi A, Yamamoto R, Ishii K, Mitsuda N. Perinatal and one-year outcomes of non-immune hydrops fetalis by etiology and age at diagnosis. J Obstet Gynaecol Res 2016;42(04):385-391

3 Bellini C, Hennekam RCM. Non-immune hydrops fetalis: a short review of etiology and pathophysiology. Am J Med Genet A 2012; 158A(03):597-605

4 Brock WW, Bradshaw WT. Congenital chylothorax: a unique presentation of nonimmune hydrops fetalis in a preterm infant. Adv Neonatal Care 2016;16(02):114-123

5 Prontera W, Jaeggi ET, Pfizenmaier M, Tassaux D, Pfister RE. Ex utero intrapartum treatment (EXIT) of severe fetal hydrothorax. Arch Dis Child Fetal Neonatal Ed 2002;86(01):F58-F60

6 Chang YL, Lien R, Wang CJ, Chang SD, Soong YK. Congenital chylothorax in three siblings. Am J Obstet Gynecol 2005; 192(06):2065-2066
7 Henry PY, Aravindan CS, Sivakumar K, Krishna HR. Extrauterine intrapartum treatment (EXIT) in bilateral primary fetal hydrothorax. Indian J Pediatr 2009;76(01):99-101

8 Eraslan S, Melekoglu R, Celik E. Extrauterine intrapartum treatment procedure in the unilateral advanced fetal hydrothorax case. Perinatal J 2015;23(01):60-64

9 Wyckoff MH, Aziz K, Escobedo MB, et al. Part 13: Neonatal Resuscitation: 2015 American Heart Association Guidelines Update for Cardiopulmonary Resuscitation and Emergency Cardiovascular Care. Circulation 2015;132(18, Suppl 2):S543-S560

10 Bhatt S, Alison BJ, Wallace EM, et al. Delaying cord clamping until ventilation onset improves cardiovascular function at birth in preterm lambs. J Physiol 2013;591(08):2113-2126

11 Hooper SB, Te Pas AB, Lang J, et al. Cardiovascular transition at birth: a physiological sequence. Pediatr Res 2015;77(05):608-614

12 Bhatt S, Polglase GR, Wallace EM, Te Pas AB, Hooper SB. Ventilation before umbilical cord clamping improves the physiological transition at birth. Front Pediatr 2014;2:113

13 Hooper SB, Polglase GR, Roehr CC. Cardiopulmonary changes with aeration of the newborn lung. Paediatr Respir Rev 2015;16(03): 147-150

14 Backes $\mathrm{CH}$, Rivera BK, Haque U, et al. Placental transfusion strategies in very preterm neonates: a systematic review and metaanalysis. Obstet Gynecol 2014;124(01):47-56

15 Aladangady N, McHugh S, Aitchison TC, Wardrop CA, Holland BM. Infants' blood volume in a controlled trial of placental transfusion at preterm delivery. Pediatrics 2006;117(01):93-98 